2021-10-07

\title{
Estimating the correlation between semi-competing risk survival endpoints
}

Sorrell, A

http://hdl.handle.net/10026.1/17366

10.1002/bimj.202000226

Biometrical Journal: journal of mathematical methods in biosciences

Wiley-VCH Verlag

All content in PEARL is protected by copyright law. Author manuscripts are made available in accordance with publisher policies. Please cite only the published version using the details provided on the item record or document. In the absence of an open licence (e.g. Creative Commons), permissions for further reuse of content should be sought from the publisher or author. 


\section{Supplementary Material for "Estimating the correlation between semi-competing risk survival endpoints" \\ By Lexy Sorrell, Yinghui Wei, Małgorzata Wojtyś and Peter Rowe}

In the supplementary material we consider the Archimedean copulas and derive their derivatives for use in the likelihood function of equation (8) in the main text. We provide results from data analysis corresponding to Section 4 in the main text in Tables S1 to S4. Table S5 details the true values for the parameters used in the simulation studies involving the Exponential, Weibull and Gompertz survival distributions with the Normal, Clayton, Frank and Gumbel copulas. Simulation results for Section 5 of the main text investigating misspecification of the copula function in each of the three scenarios are provided in Tables S6 to S20. Finally, the contour plots from Section 4 of the main text are given in Figures S1 and S2.

\section{Archimedean copulas}

For a 2-dimensional random vector $(U, V)$, uniformly distributed on the unit cube $[0,1]^{2}$ with observed values $u$ and $v$, we consider the bivariate copula

$$
C(u, v)=P(U \leq u, V \leq v) .
$$

Archimedean copulas can be written in the form,

$$
C_{\theta}(u, v)=\psi^{-1}(\psi(u)+\psi(v)),
$$

where $\theta$ is the association parameter and $\psi$ is the generator function of the Archimedean copula that depends on $\theta$, that is $\psi(u)=\psi(u ; \theta)$.

Using known formula,

$$
\frac{d f^{-1}(y)}{d y}=\frac{1}{\left.f^{\prime}(x)\right|_{x=f^{-1}(y)}},
$$

we can use the generator functions of copulas to derive the first and second derivatives to use in the likelihood functions. First, we differentiate the copula function, equation (1), by $u$, using equation (2),

$\frac{d C_{\theta}(u, v)}{d u}=\frac{d}{d u}\left(\psi^{-1}(\psi(u)+\psi(v))\right)=\frac{1}{\left.\psi^{\prime}(x)\right|_{x=\psi^{-1}(\psi(u)+\psi(v))}} \times \psi^{\prime}(u)=\frac{\psi^{\prime}(u)}{\psi^{\prime}\left(C_{\theta}(u, v)\right)}$ 
By symmetry, differentiating equation (1) by $v$,

$$
\frac{d C_{\theta}(u, v)}{d v}=\frac{\psi^{\prime}(v)}{\psi^{\prime}\left(C_{\theta}(u, v)\right)} .
$$

Finally, the second derivative of equation (1) is found by taking the derivative of equation (3) with respect to $v$,

$$
\begin{aligned}
\frac{d^{2} C_{\theta}(u, v)}{d u d v} & =\frac{d}{d v} \frac{\psi^{\prime}(u)}{\psi^{\prime}\left(C_{\theta}(u, v)\right)}=\psi^{\prime}(u) \frac{d}{d v} \frac{1}{\psi^{\prime}\left(C_{\theta}(u, v)\right)} \\
& =\psi^{\prime}(u) \frac{-1}{\left(\psi^{\prime}\left(C_{\theta}(u, v)\right)\right)^{2}} \frac{d}{d v} \psi^{\prime}\left(C_{\theta}(u, v)\right) \\
& =\frac{-\psi^{\prime}(u)}{\left(\psi^{\prime}\left(C_{\theta}(u, v)\right)\right)^{2}} \psi^{\prime \prime}\left(C_{\theta}(u, v)\right) \frac{d}{d v} C_{\theta}(u, v) \\
& =\frac{-\psi^{\prime}(u)}{\left(\psi^{\prime}\left(C_{\theta}(u, v)\right)\right)^{2}} \psi^{\prime \prime}\left(C_{\theta}(u, v)\right) \frac{\psi^{\prime}(v)}{\psi^{\prime}\left(C_{\theta}(u, v)\right)} \\
& =\frac{-\psi^{\prime}(u) \psi^{\prime}(v) \psi^{\prime \prime}\left(C_{\theta}(u, v)\right)}{\left(\psi^{\prime}\left(C_{\theta}(u, v)\right)\right)^{3}} .
\end{aligned}
$$

\section{Clayton Copula}

The generator function for the Clayton copula is given by,

$$
\psi(t)=\frac{t^{-\theta}-1}{\theta} .
$$

The first derivative of equation (6) is given by,

$$
\psi^{\prime}(t)=\frac{-\theta t^{-\theta-1}}{\theta}=-t^{-(\theta+1)} .
$$

The second derivative of equation (6), by differentiating equation (7), is given by,

$$
\psi^{\prime \prime}(t)=(\theta+1) t^{-(\theta+2)} .
$$

Substituting equations (6) and (7) into equations (3) and (4) we find the first derivatives of the Clayton copula function with respect to $u$ and $v$ to be,

$$
\begin{aligned}
& \frac{\partial C_{\theta}(u, v)}{\partial u}=\frac{-u^{-(\theta+1)}}{-C_{\theta}(u, v)^{-(\theta+1)}}=\frac{C_{\theta}(u, v)^{\theta+1}}{u^{\theta+1}}, \\
& \frac{\partial C_{\theta}(u, v)}{\partial v}=\frac{-v^{-(\theta+1)}}{-C_{\theta}(u, v)^{-(\theta+1)}}=\frac{C_{\theta}(u, v)^{\theta+1}}{v^{\theta+1}} .
\end{aligned}
$$


Substituting equations (6), (7) and (8) into equation (5), we find the second derivative of the Clayton copula function to be,

$$
\frac{\partial^{2} C_{\theta}(u, v)}{\partial u \partial v}=\frac{u^{-(\theta+1)} v^{-(\theta+1)}(\theta+1) C_{\theta}(u, v)^{-(\theta+2)}}{C_{\theta}(u, v)^{-3(\theta+1)}}=\frac{(\theta+1) C_{\theta}(u, v)^{2 \theta+1}}{u^{\theta+1} v^{\theta+1}} .
$$

\section{Frank copula}

The generator function for the Frank copula is given by,

$$
\psi(t)=-\log \left(\frac{e^{-\theta t}-1}{e^{-\theta}-1}\right) .
$$

Therefore, the first derivative of equation (12) is given by,

$$
\psi^{\prime}(t)=-\frac{-\theta e^{-\theta t}}{e^{-\theta}-1} \frac{e^{-\theta}-1}{e^{-\theta t}-1}=\frac{\theta e^{-\theta t}}{e^{-\theta t}-1}=\frac{\theta}{1-e^{\theta t}} .
$$

Using the quotient rule, the second derivative of equation (12), by differentiating equation (13), is given by,

$$
\psi^{\prime \prime}(t)=\frac{0\left(1-e^{\theta t}\right)-\left(-\theta^{2} e^{\theta t}\right)}{\left(1-e^{\theta t}\right)^{2}}=\frac{\theta^{2} e^{\theta t}}{\left(1-e^{\theta t}\right)^{2}} .
$$

Substituting equations (12) and (13) into equations (3) and (4) we find the first derivatives of the Frank copula function with respect to $u$ and $v$ to be,

$$
\begin{aligned}
& \frac{\partial C_{\theta}(u, v)}{\partial u}=\frac{\theta}{1-e^{\theta u}} \frac{1-e^{\theta C_{\theta}(u, v)}}{\theta}=\frac{1-e^{\theta C_{\theta}(u, v)}}{1-e^{\theta u}}, \\
& \frac{\partial C_{\theta}(u, v)}{\partial v}=\frac{\theta}{1-e^{\theta v}} \frac{1-e^{\theta C_{\theta}(u, v)}}{\theta}=\frac{1-e^{\theta C_{\theta}(u, v)}}{1-e^{\theta v}} .
\end{aligned}
$$

Substituting equations (12), (13) and (14) into equation (5), we find the second derivative of the Frank copula function to be,

$$
\begin{aligned}
\frac{\partial^{2} C_{\theta}(u, v)}{\partial u \partial v} & =\frac{-\theta^{2}}{\left(1-e^{\theta u}\right)\left(1-e^{\theta v}\right)} \frac{\theta^{2} e^{\theta C_{\theta}(u, v)}}{\left(1-e^{\theta C_{\theta}(u, v)}\right)^{2}} \frac{\left(1-e^{\theta C_{\theta}(u, v)}\right)^{3}}{\theta^{3}} \\
& =\frac{-\theta^{4} e^{\theta C_{\theta}(u, v)}\left(1-e^{\theta C_{\theta}}\right)}{\theta^{3}\left(1-e^{\theta u}\right)\left(1-e^{\theta v}\right)} \\
& =\frac{\theta e^{\theta C_{\theta}(u, v)}\left(e^{\theta C_{\theta}(u, v)}-1\right)}{\left(e^{\theta u}-1\right)\left(e^{\theta v}-1\right)} .
\end{aligned}
$$




\section{Gumbel copula}

The generator function for the Gumbel copula is given by the following,

$$
\psi(t)=(-\log (t))^{\theta} .
$$

The first derivative of equation (18) is given by,

$$
\psi^{\prime}(t)=\theta(-\log (t))^{\theta-1}\left(\frac{-1}{t}\right)=\frac{-\theta(-\log (t))^{\theta-1}}{t} .
$$

The second derivative of equation (18) is given by,

$$
\begin{aligned}
\psi^{\prime \prime}(t) & =\frac{\theta(\theta-1)(-\log (t))^{\theta-2}-\left(-\theta(-\log (t))^{\theta-1}\right)}{t^{2}} \\
& =\frac{\theta(-\log (t))^{\theta-2}((\theta-1)+(-\log (t)))}{t^{2}} \\
& =\frac{\theta(-\log (t))^{\theta-2}(\theta-1-\log (t))}{t^{2}} .
\end{aligned}
$$

Substituting equations (18) and (19) into equations (3) and (4) we find the first derivatives of the Gumbel copula with respect to $u$ and $v$ to be,

$$
\begin{aligned}
& \frac{\partial C_{\theta}(u, v)}{\partial u}=\frac{\theta(-\log (u))^{\theta-1} C_{\theta}(u, v)}{\theta u\left(-\log \left(C_{\theta}(u, v)\right)\right)^{\theta-1}}=\frac{C_{\theta}(u, v)(-\log (u))^{\theta-1}}{u\left(-\log \left(C_{\theta}(u, v)\right)\right)^{\theta-1}} \\
& \frac{\partial C_{\theta}(u, v)}{\partial v}=\frac{\theta(-\log (v))^{\theta-1} C_{\theta}(u, v)}{\theta v\left(-\log \left(C_{\theta}(u, v)\right)\right)^{\theta-1}}=\frac{C_{\theta}(u, v)(-\log (v))^{\theta-1}}{v\left(-\log \left(C_{\theta}(u, v)\right)\right)^{\theta-1}} .
\end{aligned}
$$

Substituting equations (18), (19) and (20) into equation (5) we find the second derivative of the Gumbel copula function to be,

$$
\begin{aligned}
\frac{\partial^{2} C_{\theta}(u, v)}{\partial u \partial v} & =\frac{-\theta^{2}(-\log (u))^{\theta-1}(-\log (v))^{\theta-1}}{u v} \frac{\theta\left(-\log \left(C_{\theta}(u, v)\right)\right)^{\theta-2}\left(\theta-1-\log \left(C_{\theta}(u, v)\right)\right)}{\left(C_{\theta}(u, v)\right)^{2}} \\
& \frac{\left(C_{\theta}(u, v)\right)^{3}}{-\theta^{3}\left(-\log \left(C_{\theta}(u, v)\right)\right)^{3 \theta-3}} \\
& =\frac{C_{\theta}(-\log (u))^{\theta-1}(-\log (v))^{\theta-1}\left(\theta-1-\log \left(C_{\theta}(u, v)\right)\right)}{u v\left(-\log \left(C_{\theta}(u, v)\right)\right)^{2 \theta-1}}
\end{aligned}
$$




\section{Tables and Figures}

Table S1: Results from renal transplant data analysis, using the Weibull survival distribution and the Normal, Clayton, Frank and Gumbel copula functions to describe the association between graft failure and death. The shape and scale parameters, $\hat{\alpha}_{1}$ and $\hat{\beta}_{1}$ for the endpoint graft failure and $\hat{\alpha}_{2}$ and $\hat{\beta}_{2}$ for the endpoint death, respectively, are given alongside estimated Spearman's rank correlation coefficient, $\hat{\rho}$. The AIC is provided for each copula model.

\begin{tabular}{|c|ccccc|c|}
\hline Method & $\hat{\alpha}_{1}(95 \% \mathrm{CI})$ & $\hat{\beta}_{1}(95 \% \mathrm{CI})$ & $\hat{\alpha}_{2}(95 \% \mathrm{CI})$ & $\hat{\beta}_{2}(95 \% \mathrm{CI})$ & $\hat{\rho}(95 \% \mathrm{CI})$ & $\mathrm{AIC}$ \\
\hline Normal & 0.610 & 0.063 & 0.864 & 0.039 & 0.450 & 4299.5 \\
& $(0.539,0.681)$ & $(0.050,0.075)$ & $(0.776,0.953)$ & $(0.030,0.049)$ & $(0.348,0.544)$ & \\
\hline Clayton & 0.633 & 0.062 & 0.864 & 0.040 & 0.678 & 4309.4 \\
& $(0.557,0.709)$ & $(0.049,0.074)$ & $(0.776,0.953)$ & $(0.031,0.050)$ & $(0.528,0.766)$ & \\
\hline Frank & 0.630 & 0.063 & 0.863 & 0.041 & 0.540 & 4300.5 \\
& $(0.555,0.704)$ & $(0.050,0.076)$ & $(0.775,0.952)$ & $(0.031,0.051)$ & $(0.411,0.640)$ & \\
\hline Gumbel & 0.600 & 0.062 & 0.841 & 0.041 & 0.301 & 4322.6 \\
& $(0.532,0.668)$ & $(0.050,0.074)$ & $(0.756,0.926)$ & $(0.032,0.051)$ & $(0.199,0.382)$ & \\
\hline
\end{tabular}


Table S2: Results from the renal transplant data analysis, using the Gompertz survival distribution and the Normal, Clayton, Frank and Gumbel copula functions to describe the association between graft failure and death. The shape and rate parameters, $\hat{\gamma}_{1}$ and $\hat{\lambda}_{1}$ for the endpoint graft failure and $\hat{\gamma}_{2}$ and $\hat{\lambda}_{2}$ for the endpoint death, respectively, are given alongside estimated Spearman's rank correlation coefficient, $\hat{\rho}$. The AIC is provided for each copula model.

\begin{tabular}{|c|ccccc|c|}
\hline Method & $\hat{\gamma}_{1}(95 \% \mathrm{CI})$ & $\hat{\lambda}_{1}(95 \% \mathrm{CI})$ & $\hat{\gamma}_{2}(95 \% \mathrm{CI})$ & $\hat{\lambda}_{2}(95 \% \mathrm{CI})$ & $\hat{\rho}(95 \% \mathrm{CI})$ & $\mathrm{AIC}$ \\
\hline Normal & -0.083 & 0.040 & -0.007 & 0.029 & 0.388 & 4364.5 \\
& $(-0.118,-0.047)$ & $(0.032,0.048)$ & $(-0.033,0.019)$ & $(0.024,0.035)$ & $(0.304,0.473)$ & \\
\hline Clayton & -0.063 & 0.039 & -0.010 & 0.032 & 0.703 & 4370.2 \\
& $(-0.099,-0.027)$ & $(0.031,0.047)$ & $(-0.036,0.015)$ & $(0.026,0.038)$ & $(0.573,0.781)$ & \\
\hline Frank & -0.069 & 0.040 & -0.010 & 0.032 & 0.559 & 4360.8 \\
& $(-0.104,-0.034)$ & $(0.032,0.048)$ & $(-0.036,0.015)$ & $(0.026,0.038)$ & $(0.441,0.651)$ & \\
\hline Gumbel & -0.091 & 0.039 & -0.004 & 0.028 & 0.217 & 4366.2 \\
& $(-0.126,-0.055)$ & $(0.032,0.047)$ & $(-0.029,0.022)$ & $(0.023,0.034)$ & $(0.137,0.287)$ & \\
\hline
\end{tabular}


Table S3: Results from the Amsterdam Cohort Study data analysis, using the Weibull survival distribution and the Normal, Clayton, Frank and Gumbel copula functions to describe the association between virus phenotype switching from non-syncytium-inducing to syncytium-inducing (SI switch) and death from AIDS. The shape and scale parameters, $\hat{\alpha}_{1}$ and $\hat{\beta}_{1}$ for the endpoint SI switch and $\hat{\alpha}_{2}$ and $\hat{\beta}_{2}$ for the endpoint death from AIDS, respectively, are given alongside estimated Spearman's rank correlation coefficient, $\hat{\rho}$. The AIC is provided for each copula model.

\begin{tabular}{|c|ccccc|c|}
\hline Method & $\hat{\alpha}_{1}(95 \% \mathrm{CI})$ & $\hat{\beta}_{1}(95 \% \mathrm{CI})$ & $\hat{\alpha}_{2}(95 \% \mathrm{CI})$ & $\hat{\beta}_{2}(95 \% \mathrm{CI})$ & $\hat{\rho}(95 \% \mathrm{CI})$ & $\mathrm{AIC}$ \\
\hline Normal & 1.208 & 0.043 & 1.957 & 0.009 & 0.511 & 2051.6 \\
& $(1.032,1.383)$ & $(0.026,0.059)$ & $(1.751,2.163)$ & $(0.005,0.013)$ & $(0.399,0.624)$ & \\
\hline Clayton & 1.125 & 0.054 & 1.952 & 0.009 & 0.639 & 2051.6 \\
& $(0.954,1.296)$ & $(0.033,0.074)$ & $(1.741,2.164)$ & $(0.005,0.013)$ & $(0.494,0.730)$ & \\
\hline Frank & 1.061 & 0.064 & 1.903 & 0.010 & 0.674 & 2048.2 \\
& $(0.901,1.221)$ & $(0.040,0.087)$ & $(1.694,2.112)$ & $(0.005,0.015)$ & $(0.564,0.752)$ & \\
\hline Gumbel & 0.914 & 0.075 & 1.933 & 0.009 & 0.414 & 2070.4 \\
& $(0.773,1.055)$ & $(0.051,0.100)$ & $(1.721,2.146)$ & $(0.005,0.014)$ & $(0.273,0.518)$ & \\
\hline
\end{tabular}


Table S4: Results from the Amsterdam Cohort Study data analysis, using the Gompertz survival distribution and the Normal, Clayton, Frank and Gumbel copula functions to describe the association between SI switch and death from AIDS. The shape and rate parameters, $\hat{\gamma}_{1}$ and $\hat{\lambda}_{1}$ for the endpoint SI switch and $\hat{\gamma}_{2}$ and $\hat{\lambda}_{2}$ for the endpoint death from AIDS, respectively, are given alongside estimated Spearman's rank correlation coefficient, $\hat{\rho}$. The AIC is provided for each copula model.

\begin{tabular}{|c|ccccc|c|}
\hline Method & $\hat{\gamma}_{1}(95 \% \mathrm{CI})$ & $\hat{\lambda}_{1}(95 \% \mathrm{CI})$ & $\hat{\gamma}_{2}(95 \% \mathrm{CI})$ & $\hat{\lambda}_{2}(95 \% \mathrm{CI})$ & $\hat{\rho}(95 \% \mathrm{CI})$ & $\mathrm{AIC}$ \\
\hline Normal & 0.054 & 0.052 & 0.228 & 0.020 & 0.567 & 2053.4 \\
& $(-0.004,0.112)$ & $(0.036,0.069)$ & $(0.182,0.274)$ & $(0.013,0.027)$ & $(0.466,0.670)$ & \\
\hline Clayton & 0.072 & 0.050 & 0.213 & 0.022 & 0.613 & 2060.5 \\
& $(0.013,0.130)$ & $(0.034,0.066)$ & $(0.167,0.259)$ & $(0.015,0.030)$ & $(0.458,0.677)$ & \\
\hline Frank & 0.054 & 0.054 & 0.218 & 0.021 & 0.583 & 2053.1 \\
& $(-0.004,0.111)$ & $(0.037,0.071)$ & $(0.171,0.264)$ & $(0.014,0.029)$ & $(0.458,0.677)$ & \\
\hline Gumbel & 0.030 & 0.056 & 0.231 & 0.019 & 0.438 & 2070.7 \\
& $(-0.027,0.087)$ & $(0.040,0.073)$ & $(0.185,0.278)$ & $(0.013,0.026)$ & $(0.314,0.534)$ & \\
\hline
\end{tabular}


Table S5: True values used for simulating data from the Exponential, Weibull and Gompertz survival distributions used to mimic the Renal transplant and Amsterdam Cohort Study data sets for the simulations investigating the effects of misspecification. Presented are values of the hazard rates of the non-terminal and terminal events, $\lambda_{1}$ and $\lambda_{2}$ for the Exponential distribution. For the Weibull distribution the shape and scale parameters are given by, $\alpha_{1}$ and $\beta_{1}$ for the non-terminal events, and $\alpha_{2}$ and $\beta_{2}$ for the terminal events, respectively. For the Gompertz distribution, the shape and rate parameters are given by $\gamma_{1}$ and $\lambda_{1}$ for the non-terminal events, and $\gamma_{2}$ and $\lambda_{2}$ for the terminal events, respectively.

\begin{tabular}{|c|c|c|c|c|c|c|c|c|c|c|c|c|c|c|}
\hline \multirow{2}{*}{ Data set } & \multirow{2}{*}{ Underlying copula } & \multicolumn{3}{|c|}{ Exponential } & \multicolumn{5}{|c|}{ Weibull } & \multicolumn{5}{|c|}{ Gompertz } \\
\hline & & $\lambda_{1}$ & $\lambda_{2}$ & $\rho$ & $\alpha_{1}$ & $\bar{\beta}$ & $\alpha_{2}$ & $\beta_{2}$ & $\rho$ & $\gamma_{1}$ & $\lambda_{1}$ & $\gamma_{2}$ & $\lambda_{2}$ & $\rho$ \\
\hline \multirow{4}{*}{ Renal transplant } & Normal & 0.03 & 0.03 & 0.39 & 0.61 & 0.06 & 0.86 & 0.04 & 0.45 & 0.001 & 0.04 & 0.001 & 0.03 & 0.39 \\
\hline & Clayton & 0.03 & 0.03 & 0.72 & 0.63 & 0.06 & 0.86 & 0.04 & 0.68 & 0.001 & 0.04 & 0.001 & 0.03 & 0.70 \\
\hline & Frank & 0.03 & 0.03 & 0.58 & 0.63 & 0.06 & 0.86 & 0.04 & 0.54 & 0.001 & 0.04 & 0.001 & 0.03 & 0.56 \\
\hline & Gumbel & 0.03 & 0.03 & 0.21 & 0.60 & 0.06 & 0.84 & 0.04 & 0.30 & 0.001 & 0.04 & 0.001 & 0.03 & 0.22 \\
\hline \multirow{4}{*}{$\begin{array}{c}\text { Amsterdam Cohort } \\
\text { Study }\end{array}$} & Normal & 0.07 & 0.07 & 0.63 & 1.21 & 0.04 & 1.96 & 0.01 & 0.51 & 0.05 & 0.05 & 0.23 & 0.02 & 0.64 \\
\hline & Clayton & 0.07 & 0.07 & 0.75 & 1.13 & 0.05 & 1.95 & 0.01 & 0.64 & 0.07 & 0.05 & 0.21 & 0.02 & 0.61 \\
\hline & Frank & 0.08 & 0.07 & 0.70 & 1.06 & 0.06 & 1.90 & 0.01 & 0.67 & 0.05 & 0.05 & 0.22 & 0.02 & 0.58 \\
\hline & Gumbel & 0.07 & 0.07 & 0.50 & 0.91 & 0.08 & 1.93 & 0.01 & 0.41 & 0.03 & 0.06 & 0.23 & 0.02 & 0.44 \\
\hline
\end{tabular}




\subsection{Misspecification of the copula function simulation results with the renal trans- plant data}

Table S6: Simulation results investigating the effect of misspecification of the copula function with assumed Spearman's rank correlation coefficient, $\rho=0$. The simulated data mimics the hazard rates for the endpoints of graft failure and death from the renal transplant data set. The data is generated from the Exponential survival distributions and the Independence copula function. The Exponential survival distribution along with the Normal, Clayton, Frank and Gumbel copula functions are used to compare the performance metrics of the hazard rates and correlation estimates under misspecification of the copula function. The number of simulated data sets is 1000 with 1199 individuals in each. MAE refers to the mean absolute error and the coverage probability is given as a percentage.

\begin{tabular}{|c|c|c|c|c|c|c|c|c|c|c|}
\hline \multirow{3}{*}{ Underlying copula } & \multicolumn{10}{|c|}{ Assumed copula distribution } \\
\hline & & \multicolumn{2}{|c|}{ Normal } & \multicolumn{2}{|c|}{ Clayton } & \multicolumn{2}{|c|}{ Frank } & \multicolumn{3}{|c|}{ Gumbel } \\
\hline & & MAE & Coverage & MAE & Coverage & MAE & Coverage & MAE & Coverage & Boundary $^{1}$ \\
\hline Independence & $\lambda_{1}$ & 0.001 & 95.9 & 0.001 & 95.5 & 0.001 & 95.9 & 0.001 & 95.9 & \\
\hline & $\lambda_{2}$ & 0.001 & 94.7 & 0.001 & 94.8 & 0.001 & 95.2 & 0.001 & 95.4 & \\
\hline & $\rho$ & 0.025 & 97.2 & 0.029 & 98.9 & 0.020 & 97.4 & 0.020 & 99.0 & 989 \\
\hline
\end{tabular}

${ }^{1}$ Boundary refers to the number of times the lower confidence interval for $\theta$ is less than or equal to 1 , the boundary for the Gumbel copula. 
Table S7: Simulation results investigating the effect of misspecification of the copula function with assumed Spearman's rank correlation coefficient, $\rho=0.25$. The simulated data mimics the hazard rates for the endpoints of graft failure and death from the renal transplant data set. The data is generated from the Exponential survival distributions and the Normal, Clayton, Frank and Gumbel copula functions. The Exponential survival distribution along with the Normal, Clayton, Frank and Gumbel copula functions are used to compare the performance metrics of the hazard rates and correlation estimates under misspecification of the copula function. The number of simulated data sets is 1000 with 1199 individuals in each. MAE refers to the mean absolute error and the coverage probability is given as a percentage.

\begin{tabular}{|c|c|c|c|c|c|c|c|c|c|c|}
\hline \multirow{3}{*}{ Underlying copula } & \multicolumn{10}{|c|}{ Assumed copula distribution } \\
\hline & & \multicolumn{2}{|c|}{ Normal } & \multicolumn{2}{|c|}{ Clayton } & \multicolumn{2}{|c|}{ Frank } & \multicolumn{3}{|c|}{ Gumbel } \\
\hline & & MAE & Coverage & MAE & Coverage & MAE & Coverage & MAE & Coverage & Boundary \\
\hline \multirow[t]{3}{*}{ Normal } & $\lambda_{1}$ & 0.001 & 95.5 & 0.001 & 93.7 & 0.001 & 94.5 & 0.001 & 92.3 & \\
\hline & $\lambda_{2}$ & 0.001 & 95.2 & 0.001 & 95.0 & 0.001 & 94.3 & 0.001 & 94.7 & \\
\hline & $\rho$ & 0.039 & 95.0 & 0.050 & 94.1 & 0.040 & 94.0 & 0.074 & 60.0 & 34 \\
\hline \multirow[t]{3}{*}{ Clayton } & $\lambda_{1}$ & 0.001 & 92.6 & 0.001 & 93.4 & 0.001 & 94.0 & 0.001 & 88.5 & \\
\hline & $\lambda_{2}$ & 0.001 & 95.3 & 0.001 & 95.8 & 0.001 & 95.2 & 0.001 & 95.21 & \\
\hline & $\rho$ & 0.066 & 77.1 & 0.047 & 94.6 & 0.060 & 80.6 & 0.140 & 12.4 & 392 \\
\hline \multirow[t]{3}{*}{ Frank } & $\lambda_{1}$ & 0.001 & 93.5 & 0.001 & 93.9 & 0.001 & 95.3 & 0.001 & 89.8 & \\
\hline & $\lambda_{2}$ & 0.001 & 93.4 & 0.001 & 94.9 & 0.001 & 95.5 & 0.001 & 94.7 & \\
\hline & $\rho$ & 0.041 & 93.6 & 0.058 & 89.2 & 0.039 & 95.1 & 0.088 & 47.5 & 68 \\
\hline \multirow[t]{3}{*}{ Gumbel } & $\lambda_{1}$ & 0.001 & 93.8 & 0.001 & 92.6 & 0.001 & 93.6 & 0.001 & 95.1 & \\
\hline & $\lambda_{2}$ & 0.001 & 94.2 & 0.001 & 95.5 & 0.001 & 94.2 & 0.001 & 94.2 & \\
\hline & $\rho$ & 0.063 & 76.8 & 0.056 & 91.8 & 0.046 & 91.4 & 0.035 & 93.8 & \\
\hline
\end{tabular}


Table S8: Simulation results investigating the effect of misspecification of the copula function with assumed Spearman's rank correlation coefficient, $\rho=0.5$. The simulated data mimics the hazard rates for the endpoints of graft failure and death from the renal transplant data set. The data is generated from the Exponential survival distributions and the Normal, Clayton, Frank and Gumbel copula functions. The Exponential survival distribution along with the Normal, Clayton, Frank and Gumbel copula functions are used to compare the performance metrics of the hazard rates and correlation estimates under misspecification of the copula function. The number of simulated data sets is 1000 with 1199 individuals in each. MAE refers to the mean absolute error and the coverage probability is given as a percentage.

\begin{tabular}{|c|c|c|c|c|c|c|c|c|c|c|}
\hline \multirow{3}{*}{ Underlying copula } & \multicolumn{10}{|c|}{ Assumed copula distribution } \\
\hline & & \multicolumn{2}{|c|}{ Normal } & \multicolumn{2}{|c|}{ Clayton } & \multicolumn{2}{|c|}{ Frank } & \multicolumn{3}{|c|}{ Gumbel } \\
\hline & & MAE & Coverage & MAE & Coverage & MAE & Coverage & MAE & Coverage & Boundary \\
\hline \multirow[t]{3}{*}{ Normal } & $\lambda_{1}$ & 0.001 & 94.7 & 0.001 & 94.0 & 0.001 & 93.9 & 0.001 & 90.9 & \\
\hline & $\lambda_{2}$ & 0.001 & 95.0 & 0.001 & 94.3 & 0.001 & 94.4 & 0.001 & 92.5 & \\
\hline & $\rho$ & 0.032 & 95.1 & 0.054 & 84.3 & 0.034 & 95.0 & 0.105 & 14.8 & \\
\hline \multirow[t]{3}{*}{ Clayton } & $\lambda_{1}$ & 0.002 & 62.7 & 0.001 & 95.4 & 0.001 & 84.1 & 0.002 & 43.9 & \\
\hline & $\lambda_{2}$ & 0.001 & 91.2 & 0.001 & 96.0 & 0.001 & 84.3 & 0.001 & 79.2 & \\
\hline & $\rho$ & 0.141 & 0.0 & 0.017 & 95.0 & 0.070 & 9.9 & 0.257 & 0.0 & \\
\hline \multirow[t]{3}{*}{ Frank } & $\lambda_{1}$ & 0.001 & 94.8 & 0.001 & 94.7 & 0.001 & 94.2 & 0.001 & 91.2 & \\
\hline & $\lambda_{2}$ & 0.001 & 94.9 & 0.001 & 95.0 & 0.001 & 95.0 & 0.001 & 95.2 & \\
\hline & $\rho$ & 0.040 & 94.5 & 0.055 & 93.0 & 0.040 & 94.6 & 0.070 & 62.8 & 356 \\
\hline \multirow[t]{3}{*}{ Gumbel } & $\lambda_{1}$ & 0.001 & 93.6 & 0.001 & 93.0 & 0.001 & 91.1 & 0.001 & 95.5 & \\
\hline & $\lambda_{2}$ & 0.001 & 94.2 & 0.001 & 95.1 & 0.001 & 91.1 & 0.001 & 94.8 & \\
\hline & $\rho$ & 0.084 & 36.3 & 0.076 & 68.9 & 0.055 & 74.9 & 0.029 & 95.5 & \\
\hline
\end{tabular}


Table S9: Simulation results investigating the effect of misspecification of the copula function with assumed Spearman's rank correlation coefficient, $\rho=0.75$. The simulated data mimics the hazard rates for the endpoints of graft failure and death from the renal transplant data set. The data is generated from the Exponential survival distributions and the Normal, Clayton, Frank and Gumbel copula functions. The Exponential survival distribution along with the Normal, Clayton, Frank and Gumbel copula functions are used to compare the performance metrics of the hazard rates and correlation estimates under misspecification of the copula function. The number of simulated data sets is 1000 with 1199 individuals in each. MAE refers to the mean absolute error and the coverage probability is given as a percentage.

\begin{tabular}{|c|c|cc|cc|cc|cc|}
\hline \multirow{3}{*}{ Underlying copula } & \multicolumn{9}{c|}{ Assumed copula distribution } \\
\cline { 2 - 10 } & & \multicolumn{2}{|c|}{ Normal } & \multicolumn{2}{c|}{ Clayton } & \multicolumn{2}{c|}{ Frank } & \multicolumn{2}{c|}{ Gumbel } \\
\cline { 2 - 10 } & & MAE & Coverage & MAE & Coverage & MAE & Coverage & MAE & Coverage \\
\hline Normal & $\lambda_{1}$ & 0.001 & 93.6 & 0.001 & 92.7 & 0.001 & 88.0 & 0.001 & 91.7 \\
& $\lambda_{2}$ & 0.001 & 94.6 & 0.001 & 95.4 & 0.001 & 91.3 & 0.001 & 91.3 \\
& $\rho$ & 0.018 & 95.0 & 0.046 & 59.4 & 0.019 & 96.2 & 0.102 & 0.9 \\
\hline Clayton & $\lambda_{1}$ & 0.001 & 71.2 & 0.001 & 96.4 & 0.001 & 87.9 & 0.002 & 57.6 \\
& $\lambda_{2}$ & 0.001 & 90.6 & 0.001 & 96.0 & 0.001 & 89.1 & 0.001 & 84.1 \\
& $\rho$ & 0.143 & 0.1 & 0.021 & 93.9 & 0.083 & 13.7 & 0.262 & 0.0 \\
\hline Frank & $\lambda_{1}$ & 0.001 & 79.4 & 0.001 & 92.2 & 0.001 & 93.8 & 0.002 & 65.4 \\
& $\lambda_{2}$ & 0.001 & 93.0 & 0.001 & 95.0 & 0.001 & 95.2 & 0.001 & 85.7 \\
& $\rho$ & 0.054 & 45.4 & 0.056 & 41.0 & 0.021 & 95.1 & 0.160 & 0.0 \\
\hline Gumbel & $\lambda_{1}$ & 0.001 & 94.3 & 0.001 & 89.5 & 0.002 & 77.6 & 0.001 & 93.0 \\
& $\lambda_{2}$ & 0.001 & 94.0 & 0.001 & 93.7 & 0.002 & 76.5 & 0.001 & 93.6 \\
& $\rho$ & 0.065 & 11.0 & 0.073 & 20.6 & 0.046 & 53.1 & 0.018 & 95.0 \\
\hline
\end{tabular}


Table S10: Simulation results investigating the effect of misspecification of the copula function with assumed Spearman's rank correlation coefficient, $\rho=0.9$. The simulated data mimics the hazard rates for the endpoints of graft failure and death from the renal transplant data set. The data is generated from the Exponential survival distributions and the Normal, Clayton, Frank and Gumbel copula functions. The Exponential survival distribution along with the Normal, Clayton, Frank and Gumbel copula functions are used to compare the performance metrics of the hazard rates and correlation estimates under misspecification of the copula function. The number of simulated data sets is 1000 with 1199 individuals in each. MAE refers to the mean absolute error and the coverage probability is given as a percentage.

\begin{tabular}{|c|c|cc|cc|cc|cc|}
\hline \multirow{3}{*}{ Underlying copula } & \multicolumn{9}{c|}{ Assumed copula distribution } \\
\cline { 2 - 10 } & & \multicolumn{2}{|c|}{ Normal } & \multicolumn{2}{c|}{ Clayton } & \multicolumn{2}{c|}{ Frank } & \multicolumn{2}{c|}{ Gumbel } \\
\cline { 2 - 10 } & & MAE & Coverage & MAE & Coverage & MAE & Coverage & MAE & Coverage \\
\hline Normal & $\lambda_{1}$ & 0.001 & 94.8 & 0.001 & 85.9 & 0.001 & 77.8 & 0.001 & 93.0 \\
& $\lambda_{2}$ & 0.001 & 94.6 & 0.001 & 94.4 & 0.001 & 87.3 & 0.001 & 92.2 \\
& $\rho$ & 0.008 & 94.2 & 0.024 & 41.4 & 0.009 & 96.1 & 0.063 & 0.0 \\
\hline Clayton & $\lambda_{1}$ & 0.002 & 39.8 & 0.001 & 94.9 & 0.001 & 78.2 & 0.003 & 22.5 \\
& $\lambda_{2}$ & 0.001 & 88.9 & 0.001 & 94.4 & 0.001 & 84.9 & 0.002 & 66.6 \\
& $\rho$ & 0.128 & 0.0 & 0.009 & 95.7 & 0.047 & 6.9 & 0.232 & 0.0 \\
\hline Frank & $\lambda_{1}$ & 0.022 & 60.5 & 0.001 & 91.8 & 0.001 & 94.6 & 0.002 & 40.6 \\
& $\lambda_{2}$ & 0.001 & 92.7 & 0.001 & 94.3 & 0.001 & 94.0 & 0.001 & 77.5 \\
& $\rho$ & 0.060 & 1.2 & 0.029 & 25.2 & 0.009 & 94.6 & 0.148 & 0.0 \\
\hline Gumbel & $\lambda_{1}$ & 0.001 & 95.3 & 0.001 & 89.1 & 0.002 & 61.9 & 0.001 & 95.1 \\
& $\lambda_{2}$ & 0.001 & 94.4 & 0.001 & 94.4 & 0.002 & 64.9 & 0.001 & 94.6 \\
& $\rho$ & 0.032 & 4.7 & 0.038 & 7.7 & 0.025 & 33.9 & 0.001 & 93.9 \\
\hline
\end{tabular}




\subsection{Misspecification of the copula function simulation results with the Amsterdam Cohort Study data}

Table S11: Simulation results investigating the effect of misspecification of the copula function with assumed Spearman's rank correlation coefficient, $\rho=0$. The simulated data mimics the hazard rates for the endpoints of SI switch and death from AIDS from the Amsterdam Cohort Study data set. The data is generated from the Exponential survival distributions and the Independence copula function. The Exponential survival distribution along with the Normal, Clayton, Frank and Gumbel copula functions are used to compare the performance metrics of the hazard rates and correlation estimates under misspecification of the copula function. The number of simulated data sets is 1000 with 329 individuals in each. MAE refers to the mean absolute error and the coverage probability is given as a percentage.

\begin{tabular}{|c|c|c|c|c|c|c|c|c|c|c|}
\hline \multirow{3}{*}{ Underlying copula } & \multicolumn{10}{|c|}{ Assumed copula distribution } \\
\hline & & \multicolumn{2}{|c|}{ Normal } & \multicolumn{2}{|c|}{ Clayton } & \multicolumn{2}{|c|}{ Frank } & \multicolumn{3}{|c|}{ Gumbel } \\
\hline & & MAE & Coverage & MAE & Coverage & MAE & Coverage & MAE & Coverage & Boundary \\
\hline \multirow[t]{3}{*}{ Independence } & $\lambda_{1}$ & 0.004 & 97.3 & 0.005 & 96.5 & 0.005 & 97.3 & 0.005 & 96.1 & \\
\hline & $\lambda_{2}$ & 0.004 & 95.8 & 0.004 & 95.4 & 0.004 & 96.1 & 0.004 & 95.1 & \\
\hline & $\rho$ & 0.039 & 97.6 & 0.042 & 98.8 & 0.036 & 97.6 & 0.031 & 99.7 & 997 \\
\hline
\end{tabular}


Table S12: Simulation results investigating the effect of misspecification of the copula function with assumed Spearman's rank correlation coefficient, $\rho=0.25$. The simulated data mimics the hazard rates for the endpoints of SI switch and death from AIDS from the Amsterdam Cohort Study data set. The data is generated from the Exponential survival distributions and the Normal, Clayton, Frank and Gumbel copula functions. The Exponential survival distribution along with the Normal, Clayton, Frank and Gumbel copula functions are used to compare the performance metrics of the hazard rates and correlation estimates under misspecification of the copula function. The number of simulated data sets is 1000 with 329 individuals in each. MAE refers to the mean absolute error and the coverage probability is given as a percentage.

\begin{tabular}{|c|c|c|c|c|c|c|c|c|c|c|}
\hline \multirow{3}{*}{ Underlying copula } & \multicolumn{10}{|c|}{ Assumed copula distribution } \\
\hline & & \multicolumn{2}{|c|}{ Normal } & \multicolumn{2}{|c|}{ Clayton } & \multicolumn{2}{|c|}{ Frank } & \multicolumn{3}{|c|}{ Gumbel } \\
\hline & & MAE & Coverage & MAE & Coverage & MAE & Coverage & MAE & Coverage & Boundary \\
\hline \multirow[t]{3}{*}{ Normal } & $\lambda_{1}$ & 0.005 & 94.4 & 0.005 & 93.9 & 0.005 & 94.2 & 0.005 & 94.2 & \\
\hline & $\lambda_{2}$ & 0.004 & 94.3 & 0.004 & 94.3 & 0.004 & 94.6 & 0.004 & 95.4 & \\
\hline & $\rho$ & 0.072 & 93.8 & 0.078 & 91.2 & 0.070 & 96.3 & 0.081 & 86.0 & 508 \\
\hline \multirow[t]{3}{*}{ Clayton } & $\lambda_{1}$ & 0.005 & 93.4 & 0.005 & 94.8 & 0.004 & 95.3 & 0.005 & 92.7 & \\
\hline & $\lambda_{2}$ & 0.004 & 93.5 & 0.004 & 95.4 & 0.004 & 94.6 & 0.004 & 94.4 & \\
\hline & $\rho$ & 0.076 & 92.4 & 0.069 & 94.4 & 0.072 & 95.1 & 0.120 & 67.6 & 759 \\
\hline \multirow[t]{3}{*}{ Frank } & $\lambda_{1}$ & 0.005 & 94.3 & 0.005 & 93.8 & 0.005 & 94.1 & 0.005 & 93.4 & \\
\hline & $\lambda_{2}$ & 0.004 & 94.2 & 0.004 & 94.5 & 0.004 & 94.0 & 0.004 & 94.9 & \\
\hline & $\rho$ & 0.072 & 94.2 & 0.084 & 90.0 & 0.072 & 94.8 & 0.093 & 82.0 & 582 \\
\hline \multirow[t]{3}{*}{ Gumbel } & $\lambda_{1}$ & 0.005 & 94.2 & 0.005 & 90.9 & 0.005 & 94.9 & 0.005 & 95.0 & \\
\hline & $\lambda_{2}$ & 0.004 & 94.4 & 0.004 & 94.1 & 0.004 & 94.8 & 0.004 & 94.4 & \\
\hline & $\rho$ & 0.083 & 88.1 & 0.093 & 85.1 & 0.076 & 93.8 & 0.066 & 94.9 & 265 \\
\hline
\end{tabular}


Table S13: Simulation results investigating the effect of misspecification of the copula function with assumed Spearman's rank correlation coefficient, $\rho=0.5$. The simulated data mimics the hazard rates for the endpoints of SI switch and death from AIDS from the Amsterdam Cohort Study data set. The data is generated from the Exponential survival distributions and the Normal, Clayton, Frank and Gumbel copula functions. The Exponential survival distribution along with the Normal, Clayton, Frank and Gumbel copula functions are used to compare the performance metrics of the hazard rates and correlation estimates under misspecification of the copula function. The number of simulated data sets is 1000 with 329 individuals in each. MAE refers to the mean absolute error and the coverage probability is given as a percentage.

\begin{tabular}{|c|c|cc|cc|cc|cc|}
\hline \multirow{3}{*}{ Underlying copula } & \multicolumn{9}{c|}{ Assumed copula distribution } \\
\cline { 2 - 10 } & & \multicolumn{2}{|c|}{ Normal } & \multicolumn{2}{c|}{ Clayton } & \multicolumn{2}{c|}{ Frank } & \multicolumn{3}{c|}{ Gumbel } \\
\cline { 2 - 10 } & & MAE & Coverage & MAE & Coverage & MAE & Coverage & MAE & Coverage \\
\hline \multirow{2}{*}{ Normal } & $\lambda_{1}$ & 0.005 & 94.8 & 0.005 & 93.3 & 0.005 & 94.6 & 0.004 & 94.1 \\
& $\lambda_{2}$ & 0.004 & 95.1 & 0.004 & 95.9 & 0.004 & 94.5 & 0.004 & 94.3 \\
& $\rho$ & 0.056 & 93.5 & 0.071 & 91.3 & 0.060 & 95.8 & 0.086 & 74.7 \\
\hline Clayton & $\lambda_{1}$ & 0.008 & 66.6 & 0.004 & 95.9 & 0.005 & 80.8 & 0.001 & 61.3 \\
& $\lambda_{2}$ & 0.004 & 92.9 & 0.004 & 95.1 & 0.005 & 86.1 & 0.005 & 90.4 \\
& $\rho$ & 0.122 & 24.4 & 0.029 & 95.1 & 0.049 & 78.1 & 0.221 & 0.3 \\
\hline Frank & $\lambda_{1}$ & 0.005 & 93.8 & 0.005 & 94.2 & 0.005 & 95.3 & 0.005 & 95.4 \\
& $\lambda_{2}$ & 0.004 & 95.4 & 0.004 & 95.2 & 0.004 & 94.9 & 0.004 & 95.6 \\
& $\rho$ & 0.072 & 96.8 & 0.074 & 96.9 & 0.069 & 97.3 & 0.081 & 91.3 \\
\hline Gumbel & $\lambda_{1}$ & 0.005 & 94.2 & 0.006 & 88.6 & 0.005 & 94.3 & 0.004 & 95.0 \\
& $\lambda_{2}$ & 0.004 & 94.5 & 0.004 & 94.9 & 0.004 & 95.2 & 0.004 & 94.3 \\
& $\rho$ & 0.074 & 81.4 & 0.091 & 83.5 & 0.066 & 92.9 & 0.057 & 94.2 \\
\hline
\end{tabular}


Table S14: Simulation results investigating the effect of misspecification of the copula function with assumed Spearman's rank correlation coefficient, $\rho=0.75$. The simulated data mimics the hazard rates for the endpoints of SI switch and death from AIDS from the Amsterdam Cohort Study data set. The data is generated from the Exponential survival distributions and the Normal, Clayton, Frank and Gumbel copula functions. The Exponential survival distribution along with the Normal, Clayton, Frank and Gumbel copula functions are used to compare the performance metrics of the hazard rates and correlation estimates under misspecification of the copula function. The number of simulated data sets is 1000 with 329 individuals in each. MAE refers to the mean absolute error and the coverage probability is given as a percentage.

\begin{tabular}{|c|c|cc|cc|cc|cc|}
\hline \multirow{3}{*}{ Underlying copula } & \multicolumn{9}{c|}{ Assumed copula distribution } \\
\cline { 2 - 10 } & & \multicolumn{2}{|c|}{ Normal } & \multicolumn{2}{c|}{ Clayton } & \multicolumn{2}{c|}{ Frank } & \multicolumn{2}{c|}{ Gumbel } \\
\cline { 2 - 10 } & & MAE & Coverage & MAE & Coverage & MAE & Coverage & MAE & Coverage \\
\hline Normal & $\lambda_{1}$ & 0.004 & 94.9 & 0.005 & 91.7 & 0.005 & 93.0 & 0.004 & 93.5 \\
& $\lambda_{2}$ & 0.004 & 95.0 & 0.004 & 94.8 & 0.004 & 95.2 & 0.004 & 93.5 \\
& $\rho$ & 0.033 & 94.2 & 0.043 & 92.9 & 0.037 & 95.5 & 0.076 & 58.1 \\
\hline Clayton & $\lambda_{1}$ & 0.007 & 74.8 & 0.004 & 94.1 & 0.005 & 85.6 & 0.007 & 67.7 \\
& $\lambda_{2}$ & 0.004 & 93.9 & 0.004 & 94.8 & 0.005 & 89.7 & 0.005 & 87.8 \\
& $\rho$ & 0.115 & 43.8 & 0.036 & 95.1 & 0.063 & 75.7 & 0.217 & 2.1 \\
\hline Frank & $\lambda_{1}$ & 0.005 & 85.8 & 0.005 & 93.6 & 0.004 & 95.1 & 0.006 & 83.2 \\
& $\lambda_{2}$ & 0.004 & 96.2 & 0.004 & 94.5 & 0.004 & 95.0 & 0.004 & 92.1 \\
& $\rho$ & 0.065 & 80.6 & 0.042 & 93.1 & 0.036 & 95.9 & 0.140 & 16.3 \\
\hline Gumbel & $\lambda_{1}$ & 0.004 & 94.3 & 0.006 & 88.2 & 0.005 & 91.9 & 0.004 & 94.3 \\
& $\lambda_{2}$ & 0.004 & 95.2 & 0.004 & 95.9 & 0.005 & 91.4 & 0.004 & 95.1 \\
& $\rho$ & 0.045 & 77.1 & 0.062 & 83.1 & 0.043 & 91.9 & 0.035 & 95.9 \\
\hline
\end{tabular}


Table S15: Simulation results investigating the effect of misspecification of the copula function with assumed Spearman's rank correlation coefficient, $\rho=0.9$. The simulated data mimics the hazard rates for the endpoints of SI switch and death from AIDS from the Amsterdam Cohort Study data set. The data is generated from the Exponential survival distributions and the Normal, Clayton, Frank and Gumbel copula functions. The Exponential survival distribution along with the Normal, Clayton, Frank and Gumbel copula functions are used to compare the performance metrics of the hazard rates and correlation estimates under misspecification of the copula function. The number of simulated data sets is 1000 with 329 individuals in each. MAE refers to the mean absolute error and the coverage probability is given as a percentage.

\begin{tabular}{|c|c|cc|cc|cc|cc|}
\hline \multirow{2}{*}{ Underlying copula } & \multicolumn{9}{c|}{ Assumed copula distribution } \\
\cline { 2 - 10 } & & \multicolumn{2}{|c|}{ Normal } & \multicolumn{2}{c|}{ Clayton } & \multicolumn{2}{c|}{ Frank } & \multicolumn{2}{c|}{ Gumbel } \\
\cline { 2 - 10 } & & MAE & Coverage & MAE & Coverage & MAE & Coverage & MAE & Coverage \\
\hline Normal & $\lambda_{1}$ & 0.004 & 95.0 & 0.005 & 89.9 & 0.005 & 89.0 & 0.004 & 95.6 \\
& $\lambda_{2}$ & 0.004 & 94.6 & 0.004 & 94.6 & 0.004 & 94.9 & 0.004 & 93.0 \\
& $\rho$ & 0.015 & 94.8 & 0.022 & 92.4 & 0.018 & 94.3 & 0.046 & 48.8 \\
\hline Clayton & $\lambda_{1}$ & 0.011 & 40.6 & 0.004 & 95.3 & 0.006 & 72.1 & 0.011 & 36.3 \\
& $\lambda_{2}$ & 0.004 & 94.0 & 0.004 & 95.5 & 0.005 & 85.1 & 0.005 & 86.2 \\
& $\rho$ & 0.127 & 3.8 & 0.017 & 95.8 & 0.039 & 66.3 & 0.217 & 0.1 \\
\hline Frank & $\lambda_{1}$ & 0.007 & 68.0 & 0.005 & 92.4 & 0.004 & 96.5 & 0.008 & 62.5 \\
& $\lambda_{2}$ & 0.004 & 95.6 & 0.004 & 94.3 & 0.004 & 94.8 & 0.004 & 91.9 \\
& $\rho$ & 0.069 & 39.0 & 0.022 & 91.9 & 0.019 & 94.1 & 0.143 & 1.2 \\
\hline Gumbel & $\lambda_{1}$ & 0.004 & 93.3 & 0.006 & 90.1 & 0.005 & 88.4 & 0.004 & 95.1 \\
& $\lambda_{2}$ & 0.004 & 94.9 & 0.004 & 94.1 & 0.005 & 88.8 & 0.004 & 94.8 \\
& $\rho$ & 0.021 & 78.7 & 0.042 & 76.2 & 0.024 & 90.0 & 0.019 & 94.4 \\
\hline
\end{tabular}




\subsection{Misspecification of the copula function with data mimicking the simulation study in Fu et al. (2013)}

Table S16: Simulation results investigating the effect of misspecification of the copula function with assumed Spearman's rank correlation coefficient, $\rho=0$. The simulated data mimics the hazard rates from the simulation study in Fu et al. (2013). The data is generated from the Exponential survival distributions and the Independence copula function. The Exponential survival distribution along with the Normal, Clayton, Frank and Gumbel copula functions are used to compare the performance metrics of the hazard rates and correlation estimates under misspecification of the copula function. The number of simulated data sets is 1000 with 100 individuals in each. MAE refers to the mean absolute error and the coverage probability is given as a percentage.

\begin{tabular}{|c|c|cc|cc|cc|ccc|}
\hline \multirow{3}{*}{ Underlying copula } & \multicolumn{9}{|c|}{ Normal } & \multicolumn{2}{c|}{ Clayton } & \multicolumn{2}{c|}{ Frank } & \multicolumn{3}{c|}{ Gumbel } \\
\cline { 2 - 12 } & & MAE & Coverage & MAE & Coverage & MAE & Coverage & MAE & Coverage & Boundary \\
\hline Independence & $\lambda_{1}$ & 0.015 & 95.6 & 0.014 & 96.4 & 0.014 & 95.2 & 0.014 & 95.7 & \\
& $\lambda_{2}$ & 0.006 & 95.2 & 0.006 & 93.8 & 0.006 & 94.8 & 0.006 & 94.8 & \\
& $\rho$ & 0.057 & 96.3 & 0.047 & 98.9 & 0.054 & 96.4 & 0.045 & 99.1 & 990 \\
\hline
\end{tabular}


Table S17: Simulation results investigating the effect of misspecification of the copula function with assumed Spearman's rank correlation coefficient, $\rho=0.25$. The simulated data mimics the hazard rates from the simulation study in $\mathrm{Fu}$ et al. (2013). The data is generated from the Exponential survival distributions and the Normal, Clayton, Frank and Gumbel copula functions. The Exponential survival distribution along with the Normal, Clayton, Frank and Gumbel copula functions are used to compare the performance metrics of the hazard rates and correlation estimates under misspecification of the copula function. The number of simulated data sets is 1000 with 100 individuals in each. MAE refers to the mean absolute error and the coverage probability is given as a percentage.

\begin{tabular}{|c|c|c|c|c|c|c|c|c|c|c|}
\hline \multirow{3}{*}{ Underlying copula } & \multicolumn{10}{|c|}{ Assumed copula distribution } \\
\hline & & \multicolumn{2}{|c|}{ Normal } & \multicolumn{2}{|c|}{ Clayton } & \multicolumn{2}{|c|}{ Frank } & \multicolumn{3}{|c|}{ Gumbel } \\
\hline & & MAE & Coverage & MAE & Coverage & MAE & Coverage & MAE & Coverage & Boundary \\
\hline \multirow[t]{3}{*}{ Normal } & $\lambda_{1}$ & 0.013 & 94.7 & 0.014 & 94.7 & 0.014 & 95.2 & 0.014 & 92.6 & \\
\hline & $\lambda_{2}$ & 0.006 & 94.8 & 0.006 & 95.2 & 0.006 & 94.7 & 0.006 & 94.4 & \\
\hline & $\rho$ & 0.099 & 94.5 & 0.103 & 91.1 & 0.094 & 97.3 & 0.102 & 91.4 & 748 \\
\hline \multirow[t]{3}{*}{ Clayton } & $\lambda_{1}$ & 0.014 & 95.4 & 0.013 & 95.5 & 0.014 & 92.4 & 0.014 & 92.6 & \\
\hline & $\lambda_{2}$ & 0.006 & 95.0 & 0.006 & 95.0 & 0.006 & 94.1 & 0.006 & 93.9 & \\
\hline & $\rho$ & 0.095 & 95.2 & 0.097 & 93.1 & 0.100 & 96.4 & 0.115 & 90.1 & 816 \\
\hline \multirow[t]{3}{*}{ Frank } & $\lambda_{1}$ & 0.013 & 95.6 & 0.014 & 94.5 & 0.013 & 96.1 & 0.014 & 94.2 & \\
\hline & $\lambda_{2}$ & 0.006 & 94.7 & 0.006 & 93.6 & 0.006 & 95.7 & 0.006 & 94.1 & \\
\hline & $\rho$ & 0.097 & 95.0 & 0.099 & 92.2 & 0.093 & 96.6 & 0.105 & 91.9 & 750 \\
\hline \multirow[t]{3}{*}{ Gumbel } & $\lambda_{1}$ & 0.014 & 95.6 & 0.014 & 95.4 & 0.014 & 95.7 & 0.014 & 94.7 & \\
\hline & $\lambda_{2}$ & 0.006 & 94.2 & 0.006 & 95.1 & 0.006 & 95.2 & 0.006 & 94.8 & \\
\hline & $\rho$ & 0.105 & 90.2 & 0.110 & 89.2 & 0.097 & 96.7 & 0.086 & 94.6 & 551 \\
\hline
\end{tabular}


Table S18: Simulation results investigating the effect of misspecification of the copula function with assumed Spearman's rank correlation coefficient, $\rho=0.5$. The simulated data mimics the hazard rates from the simulation study in Fu et al. (2013). The data is generated from the Exponential survival distributions and the Normal, Clayton, Frank and Gumbel copula functions. The Exponential survival distribution along with the Normal, Clayton, Frank and Gumbel copula functions are used to compare the performance metrics of the hazard rates and correlation estimates under misspecification of the copula function. The number of simulated data sets is 1000 with 100 individuals in each. MAE refers to the mean absolute error and the coverage probability is given as a percentage.

\begin{tabular}{|c|c|c|c|c|c|c|c|c|c|c|}
\hline \multirow{3}{*}{ Underlying copula } & \multicolumn{10}{|c|}{ Assumed copula distribution } \\
\hline & & \multicolumn{2}{|c|}{ Normal } & \multicolumn{2}{|c|}{ Clayton } & \multicolumn{2}{|c|}{ Frank } & \multicolumn{3}{|c|}{ Gumbel } \\
\hline & & MAE & Coverage & MAE & Coverage & MAE & Coverage & MAE & Coverage & Boundary \\
\hline \multirow[t]{3}{*}{ Normal } & $\lambda_{1}$ & 0.013 & 94.0 & 0.013 & 94.3 & 0.013 & 93.0 & 0.013 & 91.78 & \\
\hline & $\lambda_{2}$ & 0.006 & 94.9 & 0.006 & 93.2 & 0.006 & 93.4 & 0.006 & 93.3 & \\
\hline & $\rho$ & 0.073 & 92.4 & 0.103 & 80.9 & 0.076 & 94.4 & 0.091 & 82.7 & 51 \\
\hline \multirow[t]{3}{*}{ Clayton } & $\lambda_{1}$ & 0.013 & 94.4 & 0.012 & 96.5 & 0.015 & 80.9 & 0.013 & 94.1 & \\
\hline & $\lambda_{2}$ & 0.006 & 94.2 & 0.006 & 95.4 & 0.007 & 79.0 & 0.006 & 95.0 & \\
\hline & $\rho$ & 0.049 & 83.9 & 0.032 & 96.0 & 0.035 & 92.8 & 0.125 & 22.4 & \\
\hline \multirow[t]{3}{*}{ Frank } & $\lambda_{1}$ & 0.013 & 95.4 & 0.014 & 95.1 & 0.015 & 93.8 & 0.013 & 94.4 & \\
\hline & $\lambda_{2}$ & 0.006 & 94.1 & 0.006 & 95.1 & 0.006 & 95.9 & 0.006 & 95.6 & \\
\hline & $\rho$ & 0.094 & 95.4 & 0.095 & 98.6 & 0.094 & 97.7 & 0.095 & 98.6 & 874 \\
\hline \multirow[t]{3}{*}{ Gumbel } & $\lambda_{1}$ & 0.013 & 95.2 & 0.014 & 95.2 & 0.013 & 94.3 & 0.012 & 96.5 & \\
\hline & $\lambda_{2}$ & 0.006 & 94.7 & 0.006 & 94.4 & 0.006 & 94.8 & 0.006 & 95.6 & \\
\hline & $\rho$ & 0.083 & 83.6 & 0.100 & 84.0 & 0.074 & 94.2 & 0.068 & 93.7 & 12 \\
\hline
\end{tabular}


Table S19: Simulation results investigating the effect of misspecification of the copula function with assumed Spearman's rank correlation coefficient, $\rho=0.75$. The simulated data mimics the hazard rates from the simulation study in $\mathrm{Fu}$ et al. (2013). The data is generated from the Exponential survival distributions and the Normal, Clayton, Frank and Gumbel copula functions. The Exponential survival distribution along with the Normal, Clayton, Frank and Gumbel copula functions are used to compare the performance metrics of the hazard rates and correlation estimates under misspecification of the copula function. The number of simulated data sets is 1000 with 100 individuals in each. MAE refers to the mean absolute error and the coverage probability is given as a percentage.

\begin{tabular}{|c|c|cc|cc|cc|cc|}
\hline \multirow{2}{*}{ Underlying copula } & \multicolumn{9}{c|}{ Assumed copula distribution } \\
\cline { 2 - 10 } & & \multicolumn{2}{|c|}{ Normal } & \multicolumn{2}{c|}{ Clayton } & \multicolumn{2}{c|}{ Frank } & \multicolumn{2}{c|}{ Gumbel } \\
\cline { 2 - 10 } Normal & MAE & Coverage & MAE & Coverage & MAE & Coverage & MAE & Coverage \\
\hline & $\lambda_{1}$ & 0.012 & 95.2 & 0.012 & 94.4 & 0.012 & 93.8 & 0.012 & 95.3 \\
& $\lambda_{2}$ & 0.006 & 95.3 & 0.006 & 94.7 & 0.006 & 92.5 & 0.005 & 95.0 \\
& $\rho$ & 0.037 & 91.5 & 0.088 & 62.2 & 0.042 & 94.5 & 0.074 & 66.7 \\
\hline Clayton & $\lambda_{1}$ & 0.012 & 95.9 & 0.012 & 94.9 & 0.015 & 84.6 & 0.012 & 94.3 \\
& $\lambda_{2}$ & 0.006 & 94.6 & 0.006 & 94.9 & 0.007 & 82.4 & 0.006 & 94.6 \\
& $\rho$ & 0.054 & 89.1 & 0.041 & 94.4 & 0.048 & 92.6 & 0.134 & 34.0 \\
\hline Frank & $\lambda_{1}$ & 0.012 & 95.2 & 0.012 & 93.6 & 0.011 & 95.4 & 0.012 & 94.7 \\
& $\lambda_{2}$ & 0.006 & 95.5 & 0.006 & 94.9 & 0.006 & 95.7 & 0.006 & 94.5 \\
& $\rho$ & 0.046 & 92.0 & 0.093 & 57.5 & 0.041 & 94.8 & 0.100 & 48.5 \\
\hline Gumbel & $\lambda_{1}$ & 0.012 & 95.5 & 0.012 & 95.7 & 0.014 & 92.5 & 0.012 & 96.4 \\
& $\lambda_{2}$ & 0.006 & 94.9 & 0.006 & 94.6 & 0.006 & 92.9 & 0.006 & 95.3 \\
& $\rho$ & 0.048 & 73.6 & 0.089 & 59.4 & 0.042 & 93.4 & 0.035 & 95.1 \\
\hline
\end{tabular}


Table S20: Simulation results investigating the effect of misspecification of the copula function with assumed Spearman's rank correlation coefficient, $\rho=0.9$. The simulated data mimics the hazard rates from the simulation study in Fu et al. (2013). The data is generated from the Exponential survival distributions and the Normal, Clayton, Frank and Gumbel copula functions. The Exponential survival distribution along with the Normal, Clayton, Frank and Gumbel copula functions are used to compare the performance metrics of the hazard rates and correlation estimates under misspecification of the copula function. The number of simulated data sets is 1000 with 100 individuals in each. MAE refers to the mean absolute error and the coverage probability is given as a percentage.

\begin{tabular}{|c|c|cc|cc|cc|cc|}
\hline \multirow{3}{*}{ Underlying copula } & \multicolumn{9}{c|}{ Assumed copula distribution } \\
\cline { 2 - 11 } & & \multicolumn{2}{|c}{ Normal } & \multicolumn{2}{c|}{ Clayton } & \multicolumn{2}{c|}{ Frank } & \multicolumn{2}{c|}{ Gumbel } \\
\cline { 2 - 11 } & & MAE & Coverage & MAE & Coverage & MAE & Coverage & MAE & Coverage \\
\hline \multirow{2}{*}{ Normal } & $\lambda_{1}$ & 0.012 & 96.0 & 0.012 & 95.0 & 0.012 & 92.2 & 0.012 & 95.4 \\
& $\lambda_{2}$ & 0.006 & 95.0 & 0.006 & 94.7 & 0.006 & 92.9 & 0.006 & 93.8 \\
& $\rho$ & 0.014 & 93.6 & 0.056 & 43.6 & 0.017 & 94.1 & 0.040 & 49.5 \\
\hline Clayton & $\lambda_{1}$ & 0.014 & 93.5 & 0.012 & 94.7 & 0.018 & 72.0 & 0.013 & 92.8 \\
& $\lambda_{2}$ & 0.006 & 94.7 & 0.005 & 95.3 & 0.009 & 69.1 & 0.006 & 92.0 \\
& $\rho$ & 0.040 & 72.2 & 0.019 & 95.8 & 0.020 & 91.2 & 0.105 & 8.6 \\
\hline Frank & $\lambda_{1}$ & 0.013 & 94.8 & 0.012 & 94.5 & 0.011 & 95.2 & 0.012 & 95.5 \\
& $\lambda_{2}$ & 0.006 & 95.8 & 0.006 & 94.0 & 0.005 & 94.6 & 0.006 & 94.8 \\
& $\rho$ & 0.029 & 84.7 & 0.069 & 34.2 & 0.016 & 95.3 & 0.074 & 21.4 \\
\hline Gumbel & $\lambda_{1}$ & 0.012 & 94.8 & 0.012 & 95.4 & 0.015 & 89.5 & 0.011 & 95.7 \\
& $\lambda_{2}$ & 0.054 & 94.9 & 0.006 & 95.1 & 0.007 & 89.5 & 0.005 & 95.4 \\
& $\rho$ & 0.020 & 69.4 & 0.061 & 42.3 & 0.018 & 91.1 & 0.014 & 94.7 \\
\hline
\end{tabular}



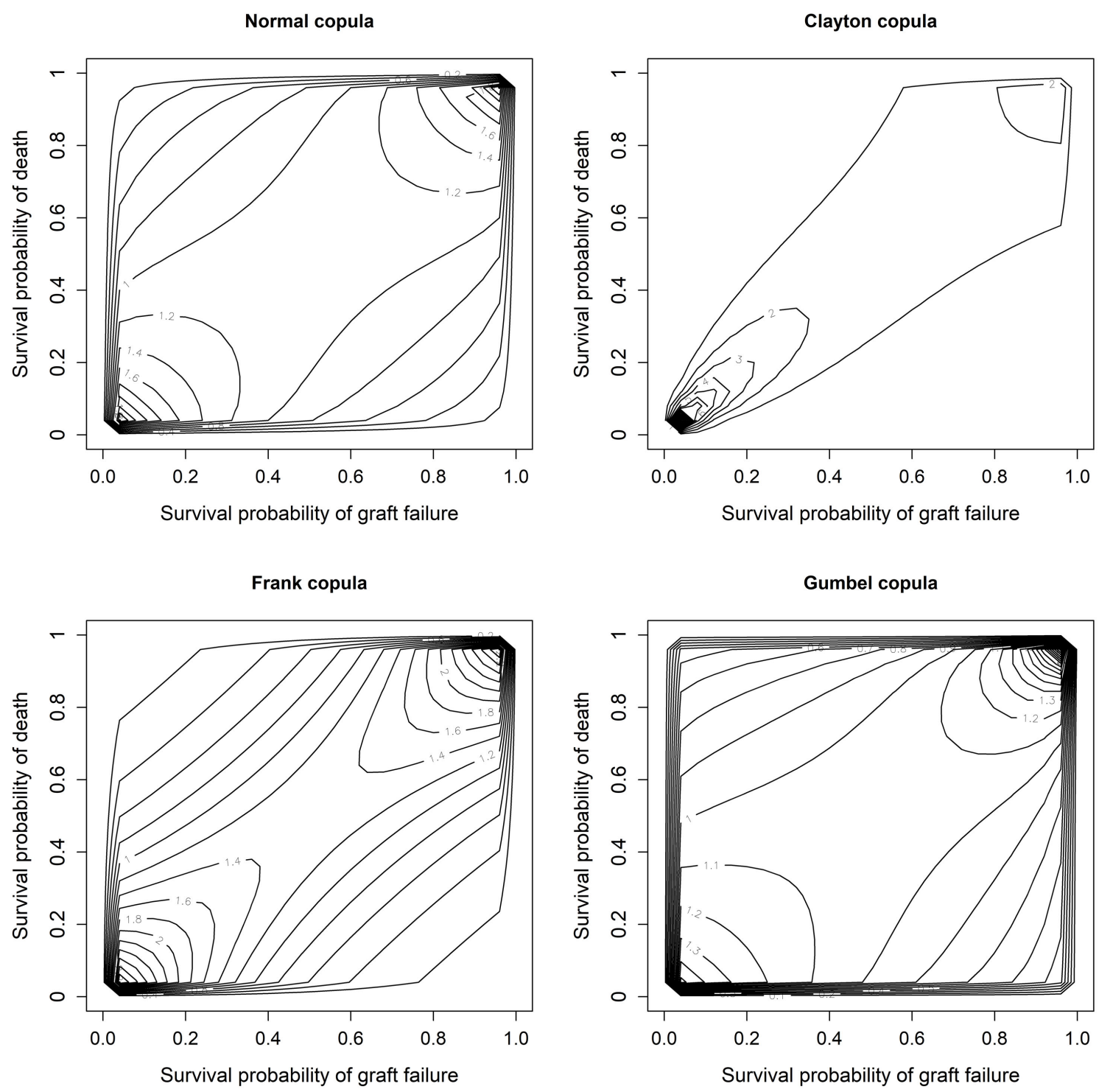

Figure S1: Normal, Clayton, Frank and Gumbel contour plots with their estimated association parameter from Table 3 of the main text, for the renal transplant data set. 

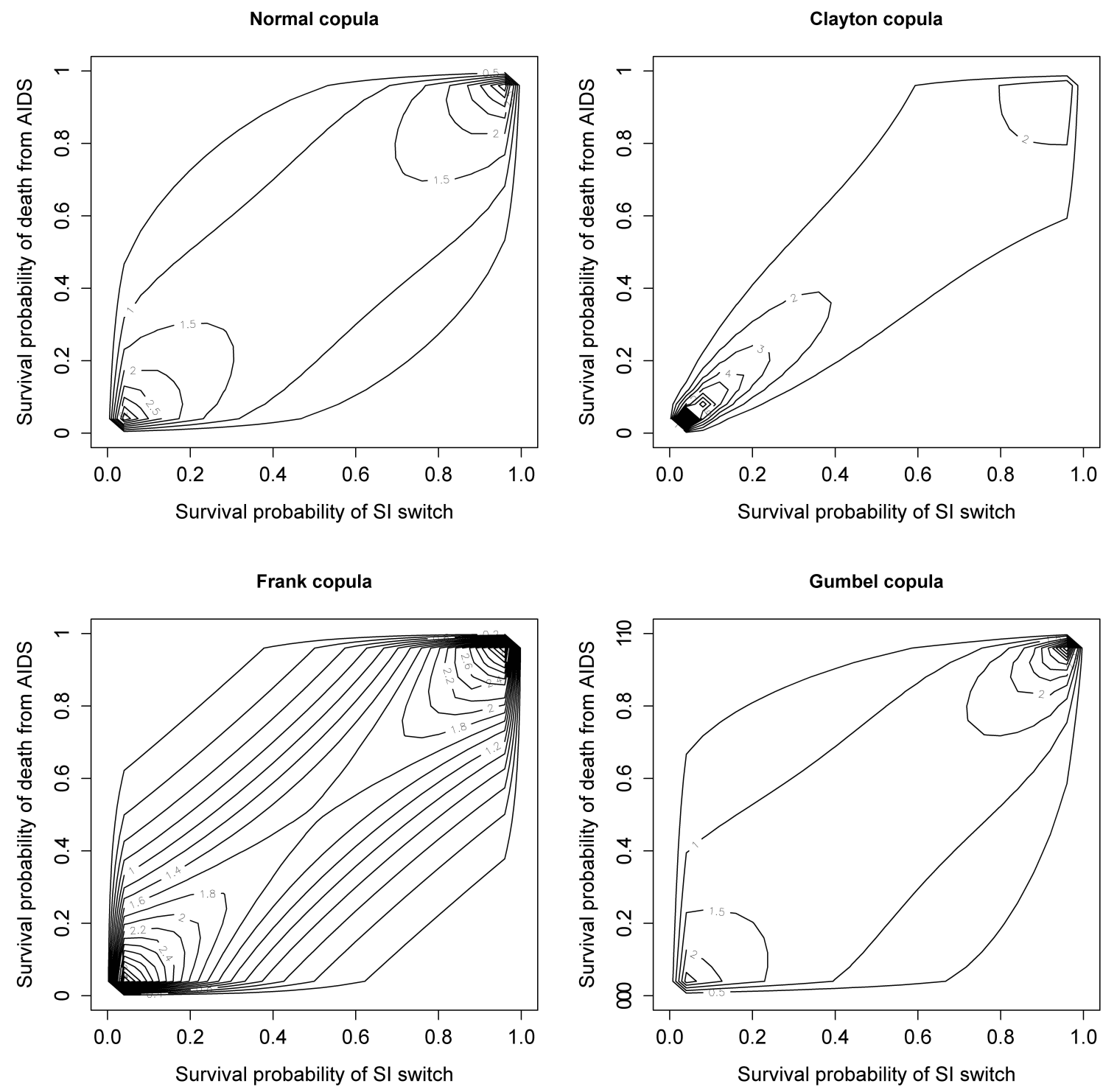

Figure S2: Normal, Clayton, Frank and Gumbel contour plots with their estimated association parameter from Table 4 of the main text, for the Amsterdam Cohort Study data set. 


\section{References}

Fu, H., Wang, Y., Liu, J., Kulkarni, P., and Melemed, A. (2013). Joint modeling of progression-free survival and overall survival by a Bayesian normal induced copula estimation model. Statistics Medicine, 32:240-254. 